\title{
In-vitro Evaluation of Different Fungicides and Bioagents against Fusarium oxysporum f. sp. lycopersici
}

\author{
A. D. Gadhave*, P. D. Patil, M. B. Dawale, A. P. Suryawnshi, M. S. Joshi and V. V. Giri \\ Department of Plant Pathology, College of Agriculture,, Dr. Balasaheb Sawant Konkan \\ Krishi Vidyapeeth, Dapoli- 415712, Ratnagiri (M.S.), India \\ *Corresponding author
}

A B S T R A C T

\begin{tabular}{|c|c|}
\hline $\begin{array}{l}\text { Ke y w o r d s } \\
\text { Fusarium } \\
\text { oxysporum f. sp. } \\
\text { lycopersici, wilt, } \\
\text { Tomato, } \\
\text { Fungicides, } \\
\text { bioagents }\end{array}$ & $\begin{array}{l}\text { Fusarium wilt of tomato incited by Fusarium oxysporum f. sp. lycopersici is one } \\
\text { of the biotic threats in profitable cultivation of tomato crop worldwide. Utilization } \\
\text { of biocontrol agents along with fungicides is best suited for integrated disease } \\
\text { management. The fungicides evaluated in vitro against Fusarium oxysporum f. sp. } \\
\text { lycopersici were effective and reduced the mycelial growth significantly. Among } \\
\text { that Carbendazim } 50 \% \mathrm{WP} \text {, Copper oxychloride } 50 \% \text { WP and Carbendazim } 25 \%\end{array}$ \\
\hline Article Info & inhibition $(100 \%),(65.22 \%)$ and $(100 \%)$ respectivly. Antagonist tested against \\
\hline $\begin{array}{l}\text { Accepted: } \\
\text { 26 July } 2020 \\
\text { Available Online: } \\
10 \text { August } 2020\end{array}$ & $\begin{array}{l}\text { Fusarium oxysporum t. sp. lycopersici in vitro signiticantly reduced the growth of } \\
\text { test pathogen. Among that Trichoderma harzianum inhibited }(52.33 \%) \text { and } \\
\text { Trichoderma virens }(49.41 \%) \text { found to be most effective with highest mycelial } \\
\text { growth. }\end{array}$ \\
\hline
\end{tabular}

\section{Introduction}

Tomato (Lycopersicon esculentum Mill.) is one of the most important vegetable crops belonging to family Solanaceae. It is supposed to be originated from Peru, (SouthAmerica). Tomato is intensively cultivated in India.

It requires moderately cool weather and is grown in both Kharif as well as Rabi seasons. It is grown round the year on variety of soils with moderate summer temperatures, well drained sandy loam soils with neutral reactions are most suitable.

Annual tomato production of India was 22337.29MT during 2017-18 with an area of about 801 thousand ha, and productivity of 27.8 MT/ha (Anonymous, 2017), It is grown in Maharashtra on an area of about 43.64 thousand ha with production of 976.58.MT, and productivity of 22.07 MT/ha (Anonymous, 2017)

Tomato crop is succumbed to Fusarium wilt at all the stages of crop growth. Pathogen incites root, stem, leaves and fruit under 
favorable condition cause drying off of the twigs and complete wilting of plants thereby resulting heavy losses. Keeping in view the economic importance of tomato, as a vegetable crop and losses incurred by Fusarium wilt in tomato, present investigations were carried out.

\section{Materials and Methods \\ Evaluation of fungicides}

Fungicides reported in Table 1, Table 2 and Table 3 were effective against Fusarium oxysporum causing wilt in tomato were evaluated in vitro by applying poisoned food technique using Potato dextrose agar as basal medium. An appropriate quantity of the fungicides was added in previously sterilized $100 \mathrm{ml}$ PDA separately in $250 \mathrm{ml}$ conical flasks. The flasks were shaken well to ensure uniform distribution of fungicides in the basal medium.

Twenty $\mathrm{ml}$ of the medium containing fungicides was poured into sterilized petri dishes. After solidification, the plates were inoculated by the fungal disc of $5 \mathrm{~mm}$ diameter cut out from seven days old culture and incubated at $27 \pm 2{ }^{\circ} \mathrm{C}$ for seven days. Observation on radial mycelia growth was recorded in all the replicated treatments. Per cent inhibition of the growth of the test pathogen was calculated by applying the formula given by Vincent (1927) and the data obtained were averaged and analyzed statistically.

Per cent Inhibition $(\mathrm{I})=\frac{\mathrm{C}-\mathrm{T}}{\mathrm{C}} \times 100$

Where,

$\mathrm{C}=$ growth $(\mathrm{mm})$ of test fungus in untreated control plate.

$\mathrm{T}=$ growth $(\mathrm{mm})$ of test fungus in treated plate.

\section{Evaluation of bio-control agents}

Six antagonist's listed in Table 4 evaluated in vitro against Fusarium oxysporum f. sp. lycopersici by dual culture method (Dennis and Webster, 1971). All antagonist's and the pathogen were multiplied in PDA. Twenty ml of PDA was poured aseptically in each petri plates and allowed to solidify. Mycelial disc of $5 \mathrm{~mm}$ diameter of each antagonist and test fungus was placed on opposite ends of PDA containing petri plates. Each treatment was replicated three times. The plates were incubated at $27 \pm 2{ }^{\circ} \mathrm{C}$ for seven days. Observation on radial mycelia growth was recorded in all the replicated treatments. Per cent inhibition of the growth of the test pathogen was calculated by applying the formula. The data obtained were averaged and analyzed statistically.

Per cent Inhibition (I) $=\frac{\mathrm{C}-\mathrm{T}}{\mathrm{C}} \times 100$

Where,

$\mathrm{C}=$ growth $(\mathrm{mm})$ of test fungus in untreated control plate.

$\mathrm{T}=$ growth $(\mathrm{mm})$ of test fungus in treated control plate.

\section{Results and Discussion}

\section{Evaluation of fungicides}

Evaluation of systemic fungicides against F. oxysporum f. sp. lycopersici

All of the six systemic fungicides (Table 4) evaluated in vitro (each at 500, 750 and 1000 $\mathrm{ppm}$ ) were found fungistatic and significantly inhibited mycelial growth of $F$. oxysporum $\mathrm{f}$. sp. lycopersici, at all three test concentrations, over untreated control.

At 500 ppm, mycelial growth inhibition of $F$. oxysporum f. sp. lycopersici ranged from 31.29 to 100.00 per cent. However, 
significantly highest and cent per cent mycelial growth inhibition (100\%) was recorded with the fungicides viz., Carbendazim 50\% WP, followed by Carboxin 75\% WP (88.44\%), Thiophanate methyl 70\% WP (88.14\%), Benomyl 50\% WP (88.14\%), Difenconazole $25 \%$ EC (72.59\%), The fungicide Azoxystrobin 23\% EC was found ineffective with $(31.29 \%)$ mycelial growth inhibition.

At $750 \mathrm{ppm}$, mycelial growth inhibition of $F$. oxysporum f. sp. lycopersici ranged from 45 to 100.00 per cent. However, significantly highest and cent per cent mycelial inhibition $(100 \%)$ was recorded with the fungicides viz., Carbendazim 50\% WP, followed by Carboxin 75\% WP (88.70\%), Thiophanate methyl 70\% WP (88.29\%), Benomyl 50\%WP (88.22\%),
Difenconazole $25 \% \quad$ EC $\quad(82.37 \%)$ and Azoxystrobin 23\% EC (45\%) which was least effective.

At $1000 \mathrm{ppm}$, fungicides tested exhibited similar trend but with increased mycelial growth inhibition as compared to that of at $500 \mathrm{ppm}$ and $750 \mathrm{ppm}$ and it was ranged from 52.77 to 100.00 per cent, However, significantly highest and cent per cent mycelial growth inhibition (100\%) was recorded with the fungicides viz., Carbendazim 50\% WP, followed by Carboxin 75\% WP (88.81), Thiophanate methyl 70\% WP (88.70), Benomyl 50\%WP (88.51), Difenconazole $25 \%$ EC (83.33), and Azoxystrobin 23\% EC (52.77\%), which was least effective (Fig. 1-3).

Table.1 List of fungicides used to check their efficacy against $F$. oxysporum f. sp. Lycopersici Systemic fungicides (each @ 500,750, and 1000 ppm)

\begin{tabular}{|l|l|c|c|}
\hline Tr. No. & Treatments & Tr. No. & Treatments \\
\hline $\mathbf{T}_{\mathbf{1}}$ & Carbendazim 50\% WP & $\mathrm{T}_{5}$ & Penconazole 10\% EC \\
\hline $\mathbf{T}_{\mathbf{2}}$ & Thiophinate methyl 70\% WP & $\mathrm{T}_{6}$ & Benomyl 50\%WP \\
\hline $\mathbf{T}_{\mathbf{3}}$ & Difenconazole 25\% EC & $\mathrm{T}_{7}$ & Carboxin 75\% WP \\
\hline $\mathbf{T}_{\mathbf{4}}$ & Azoxystrobin 23\% EC & $\mathrm{T}_{8}$ & Control (untreated) \\
\hline
\end{tabular}

Table.2 List of fungicides used to check their efficacy against $F$. oxysporum $\mathrm{f}$. sp. lycopersici. Contact and combi - fungicides (each @ 1500, 2000 and 2500 ppm)

\begin{tabular}{|l|l|l|l|}
\hline Tr. No. & Treatments & Tr. No. & Treatments \\
\hline $\mathbf{T}_{\mathbf{1}}$ & Captan 75\% WP & $\mathrm{T}_{5}$ & Carbendazim 25\% + Mancozeb 50\% WS \\
\hline $\mathbf{T}_{\mathbf{2}}$ & Thiram 75\% WS & $\mathrm{T}_{6}$ & Carboxin 37.5\% + Thiram 37.5\% WS \\
\hline $\mathbf{T}_{\mathbf{3}}$ & Copper oxychloride 50\% WP & $\mathrm{T}_{7}$ & Metalaxyl 8\% + Mancozeb 64\% WP \\
\hline $\mathbf{T}_{\mathbf{4}}$ & Mancozeb 75\% WP & $\mathrm{T}_{8}$ & Control (untreated) \\
\hline
\end{tabular}

Table.3 List of bioagents used to check their efficacy against $F$. oxysporum f. sp. Lycopersici

\begin{tabular}{|c|l|c|l|}
\hline Tr. No. & \multicolumn{1}{|c|}{ Treatments } & Tr. No. & \multicolumn{1}{|c|}{ Treatments } \\
\hline $\mathbf{T}_{\mathbf{1}}$ & T. harzianum & $\mathrm{T}_{5}$ & T. hamatum \\
\hline $\mathbf{T}_{\mathbf{2}}$ & T. viride & $\mathrm{T}_{6}$ & Pseudomonas fluorescens \\
\hline $\mathbf{T}_{\mathbf{3}}$ & T. virens & $\mathrm{T}_{7}$ & Control (Untreated) \\
\hline $\mathbf{T}_{\mathbf{4}}$ & T. koningii & & \\
\hline
\end{tabular}


Table.4 In vitro efficacy of various systemic fungicides against F.oxysporum f. sp. Lycopersici

\begin{tabular}{|c|c|c|c|c|c|c|c|c|c|}
\hline \multirow[t]{2}{*}{$\begin{array}{l}\text { Tr. } \\
\text { No. }\end{array}$} & \multirow[t]{2}{*}{ Treatment } & \multicolumn{3}{|c|}{$\begin{array}{c}\text { Colony Dia.*(mm) at } \\
\text { ppm }\end{array}$} & \multirow{2}{*}{$\begin{array}{c}\text { Av. } \\
\text { Colony } \\
(\mathbf{m m})\end{array}$} & \multicolumn{3}{|c|}{$\begin{array}{l}\% \text { Inhibition* } \\
\text { at ppm }\end{array}$} & \multirow{2}{*}{$\begin{array}{c}\text { Av. } \\
\text { Inhibitio } \\
\text { n (\%) }\end{array}$} \\
\hline & & 500 & 750 & 1000 & & 500 & 750 & 1000 & \\
\hline T1 & $\begin{array}{c}\text { Carbendazim } 50 \% \\
\text { WP }\end{array}$ & 00.00 & 00.00 & 00.00 & 00.00 & $\begin{array}{l}100.00 \\
(90.00)\end{array}$ & $\begin{array}{l}100.00 \\
(90.00)\end{array}$ & $\begin{array}{l}100.00 \\
(90.00)\end{array}$ & $\begin{array}{l}100.00 \\
(90.00)\end{array}$ \\
\hline $\mathbf{T} 2$ & $\begin{array}{c}\text { Thiophinate methyl } \\
70 \% \mathrm{WP}\end{array}$ & 10.66 & 10.53 & 10.16 & 10.45 & $\begin{array}{c}88.14 \\
(69.85)\end{array}$ & $\begin{array}{c}88.29 \\
(69.98)\end{array}$ & $\begin{array}{c}88.70 \\
(70.35)\end{array}$ & $\begin{array}{c}88.37 \\
(70.06)\end{array}$ \\
\hline T3 & $\begin{array}{c}\text { Difenconazole } 25 \% \\
\text { EC }\end{array}$ & 24.66 & 15.86 & 15 & 18.50 & $\begin{array}{c}72.59 \\
(58.42)\end{array}$ & $\begin{array}{c}82.37 \\
(65.17)\end{array}$ & $\begin{array}{c}83.33 \\
(65.90)\end{array}$ & $\begin{array}{c}79.43 \\
(63.02)\end{array}$ \\
\hline $\mathbf{T 4}$ & $\begin{array}{c}\text { Azoxystrobin } 23 \% \\
\text { EC }\end{array}$ & 61.83 & 49.5 & 42.5 & 51.27 & $\begin{array}{c}31.29 \\
(34.01)\end{array}$ & $\begin{array}{c}45.00 \\
(58.42)\end{array}$ & $\begin{array}{c}52.77 \\
(46.58)\end{array}$ & $\begin{array}{c}43.02 \\
(40.98)\end{array}$ \\
\hline T5 & Benomyl 50\%WP & 10.66 & 10.6 & 10.33 & 10.53 & $\begin{array}{c}88.14 \\
(69.85)\end{array}$ & $\begin{array}{c}88.22 \\
(34.01)\end{array}$ & $\begin{array}{c}88.51 \\
(70.18)\end{array}$ & $\begin{array}{c}88.29 \\
(69.98)\end{array}$ \\
\hline T6 & Carboxin $75 \%$ WP & 10.4 & 10.16 & 10.33 & 10.29 & $\begin{array}{c}88.44 \\
(70.12)\end{array}$ & $\begin{array}{c}88.70 \\
(69.85)\end{array}$ & $\begin{array}{c}88.81 \\
(70.45)\end{array}$ & $\begin{array}{c}88.65 \\
(70.31)\end{array}$ \\
\hline T7 & Control (untreated) & 90.00 & 90.00 & 90.00 & 90.00 & $\begin{array}{c}00.00 \\
(00.00)\end{array}$ & $\begin{array}{c}00.00 \\
(00.00)\end{array}$ & $\begin{array}{c}00.00 \\
(00.00)\end{array}$ & $\begin{array}{c}00.00 \\
(00.00)\end{array}$ \\
\hline & S. E. $\mathbf{m} \pm$ & 0.27 & 0.23 & 0.16 & - & & & & - \\
\hline & C. D. at (p 0.01) & 1.16 & 0.99 & 0.70 & - & & & & - \\
\hline
\end{tabular}

* Mean of three replications. Figures in parentheses are Arcsine values

Table.5 In vitro efficacy of contact and combi fungicides against $F$. oxysporum f. sp. Lycopersici

\begin{tabular}{|c|c|c|c|c|c|c|c|c|c|}
\hline \multirow[t]{2}{*}{$\begin{array}{l}\text { Tr. } \\
\text { No. }\end{array}$} & \multirow[t]{2}{*}{ Treatment } & \multicolumn{3}{|c|}{$\begin{array}{c}\text { Colony Dia.*(mm) at } \\
\text { ppm }\end{array}$} & \multirow{2}{*}{$\begin{array}{c}\text { Av. } \\
\text { Colony } \\
(\mathbf{m m})\end{array}$} & \multicolumn{3}{|c|}{$\begin{array}{l}\text { \% Inhibition* } \\
\text { at ppm }\end{array}$} & \multirow{2}{*}{$\begin{array}{c}\text { Av. } \\
\text { Inhibition } \\
(\%)\end{array}$} \\
\hline & & 1500 & 2000 & 2500 & & 1500 & 2000 & 2500 & \\
\hline T1 & Captan 75\% WP & 39.70 & 33.37 & 32.38 & 35.15 & $\begin{array}{c}55.88 \\
(48.37)\end{array}$ & $\begin{array}{c}62.92 \\
(52.48)\end{array}$ & $\begin{array}{c}64.01 \\
(53.13)\end{array}$ & $\begin{array}{c}60.93 \\
(51.31)\end{array}$ \\
\hline $\mathbf{T} 2$ & Thiram $75 \%$ WS & 37.06 & 34.71 & 32.70 & 34.82 & $\begin{array}{c}58.82 \\
(50.08)\end{array}$ & $\begin{array}{c}61.48 \\
(51.63)\end{array}$ & $\begin{array}{c}63.66 \\
(52.92)\end{array}$ & $\begin{array}{c}61.32 \\
(51.54)\end{array}$ \\
\hline T3 & $\begin{array}{c}\text { Copper oxychloride } \\
50 \% \text { WP }\end{array}$ & 34.36 & 30.7 & 29.36 & 31.47 & $\begin{array}{c}61.82 \\
(51.83)\end{array}$ & $\begin{array}{c}66.48 \\
(54.62)\end{array}$ & $\begin{array}{c}67.37 \\
(55.16)\end{array}$ & $\begin{array}{c}65.22 \\
(53.86)\end{array}$ \\
\hline T4 & Mancozeb 75\% WP & 66.02 & 47.70 & 47.05 & 53.59 & $\begin{array}{c}26.64 \\
(31.07)\end{array}$ & $\begin{array}{c}47.00 \\
(43.28)\end{array}$ & $\begin{array}{c}47.72 \\
(43.69)\end{array}$ & $\begin{array}{l}40.45 \\
(39.49)\end{array}$ \\
\hline T5 & $\begin{array}{l}\text { Carbendazim 25\% }+ \\
\text { Mancozeb } 50 \% \text { WS }\end{array}$ & 0.00 & 0.00 & 0.00 & 0.00 & $\begin{array}{l}100.00 \\
(90.00)\end{array}$ & $\begin{array}{l}100.00 \\
(90.00)\end{array}$ & $\begin{array}{l}100.00 \\
(90.00)\end{array}$ & $\begin{array}{l}100.00 \\
(90.00)\end{array}$ \\
\hline T6 & $\begin{array}{l}\text { Carboxin } 37.5 \% \\
+ \text { Thiram } 37.5 \% \mathrm{WS}\end{array}$ & 15.00 & 12.00 & 10.33 & 12.44 & $\begin{array}{c}83.33 \\
(65.90)\end{array}$ & $\begin{array}{c}86.66 \\
(68.57)\end{array}$ & $\begin{array}{c}88.51 \\
(70.18)\end{array}$ & $\begin{array}{c}86.16 \\
(68.15)\end{array}$ \\
\hline $\mathbf{T} 7$ & $\begin{array}{c}\text { Metalaxyl 8\% + } \\
\text { Mancozeb 64\% WP }\end{array}$ & 65.03 & 55.03 & 35.02 & 51.69 & $\begin{array}{c}27.74 \\
(31.78)\end{array}$ & $\begin{array}{l}38.85 \\
(35.55)\end{array}$ & $\begin{array}{c}61.09 \\
(51.40)\end{array}$ & $\begin{array}{c}42.56 \\
(40.72)\end{array}$ \\
\hline T8 & Control (untreated) & 90.00 & 90.00 & 90.00 & 90.00 & $\begin{array}{l}100.00 \\
(90.00)\end{array}$ & $\begin{array}{l}100.00 \\
(90.00)\end{array}$ & $\begin{array}{l}100.00 \\
(90.00)\end{array}$ & $\begin{array}{l}100.00 \\
(90.00)\end{array}$ \\
\hline & S. E. $\mathbf{m} \pm$ & 0.16 & 0.20 & 0.24 & - & & & & - \\
\hline & C. D. at (p 0.01) & 0.68 & 0.83 & 0.98 & - & & & & - \\
\hline
\end{tabular}

* Mean of three replications. Figures in parentheses are Arcsine values 
Table.6 In vitro efficacy of different bioagents against $F$. oxysporum f. sp. lycopersici

\begin{tabular}{|c|c|c|c|}
\hline Tr. No. & Treatments & $\begin{array}{c}\text { Mean Colony } \\
\text { Diameter }(\mathbf{m m}) *\end{array}$ & $\begin{array}{l}\text { Percent Inhibition } \\
\text { Over control }\end{array}$ \\
\hline T1 & Trichoderma harzianum & 42.90 & $\begin{array}{c}52.33 \\
(46.33)\end{array}$ \\
\hline $\mathbf{T} 2$ & T. viride & 60.83 & $\begin{array}{c}32.41 \\
(34.70)\end{array}$ \\
\hline T3 & T. virens & 45.53 & $\begin{array}{c}49.41 \\
(44.66)\end{array}$ \\
\hline T4 & T. koningii & 53.50 & $\begin{array}{c}40.55 \\
(39.55)\end{array}$ \\
\hline T5 & T. hamatum & 64.67 & $\begin{array}{c}28.14 \\
(32.03)\end{array}$ \\
\hline T6 & Pseudomonas fluorescens & 70.50 & $\begin{array}{c}21.66 \\
(27.73)\end{array}$ \\
\hline T7 & Control (Untreated) & 90.00 & $\begin{array}{c}00.00 \\
(00.00)\end{array}$ \\
\hline \multicolumn{2}{|c|}{ S. E. $\mathbf{m} \pm$} & \multicolumn{2}{|c|}{0.22} \\
\hline \multicolumn{2}{|c|}{ C.D $(P=0.01)$} & \multicolumn{2}{|c|}{0.96} \\
\hline
\end{tabular}

* Mean of three replications

Figures in parentheses are Arcsine values

Fig.1

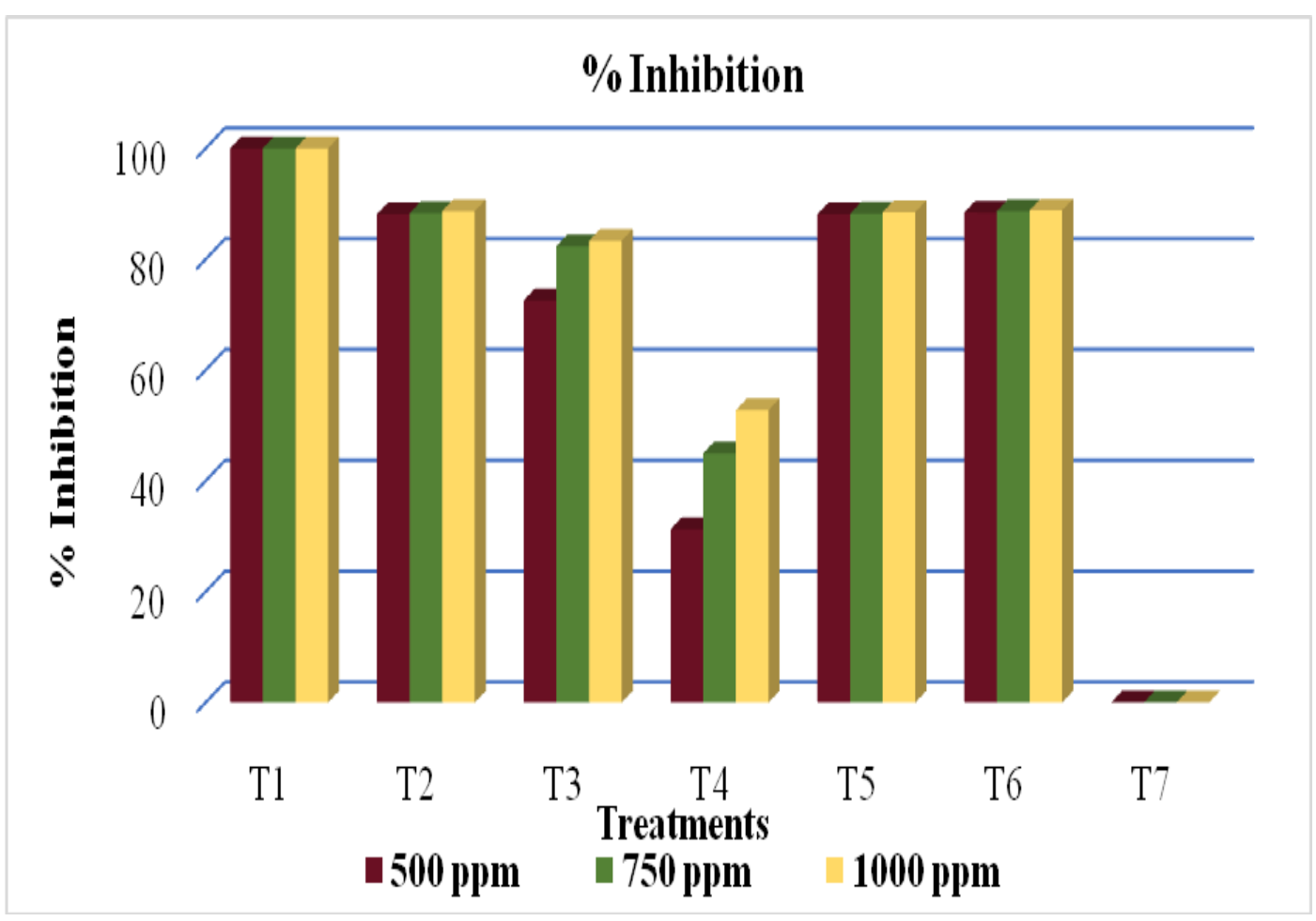


Fig.2

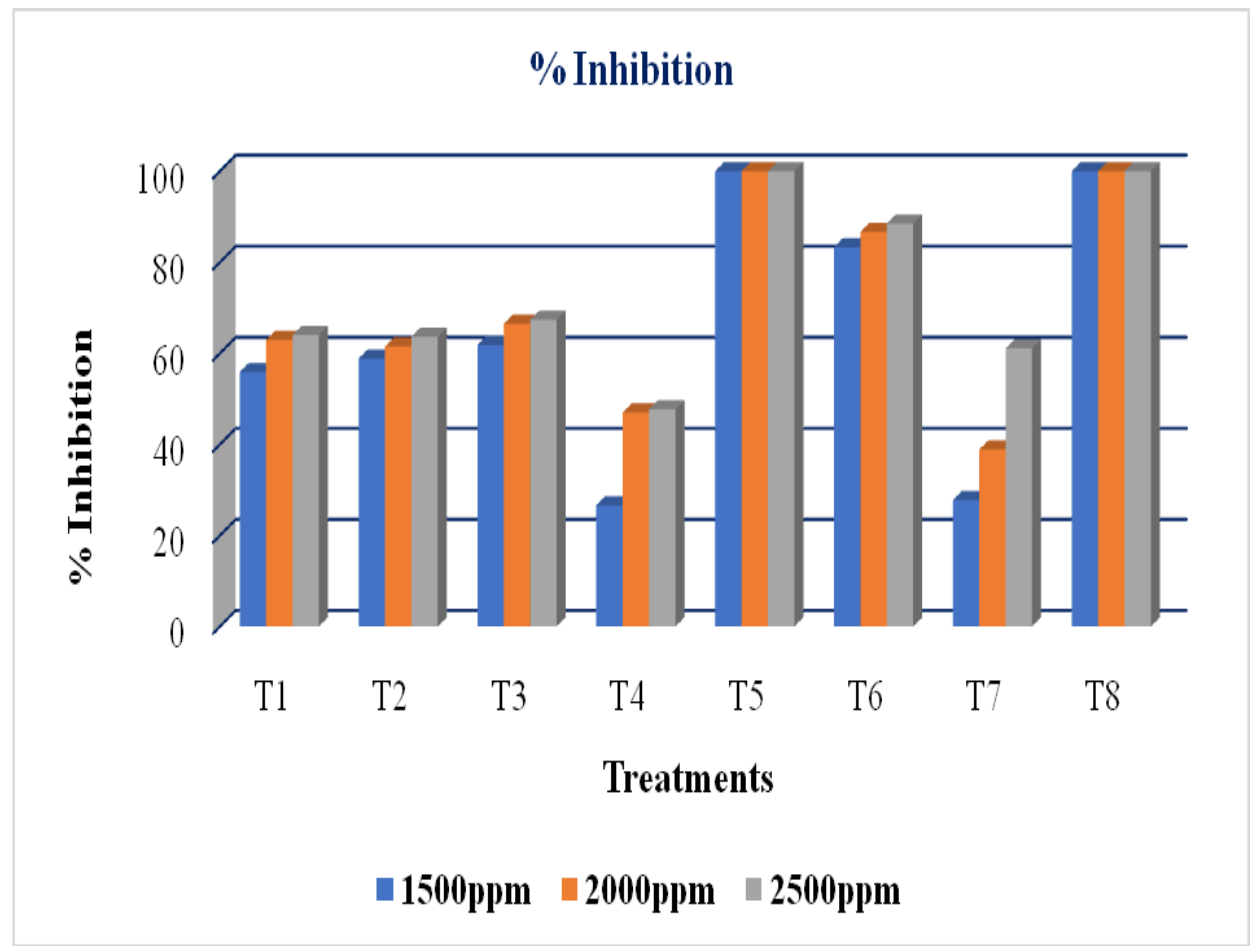

Fig.3

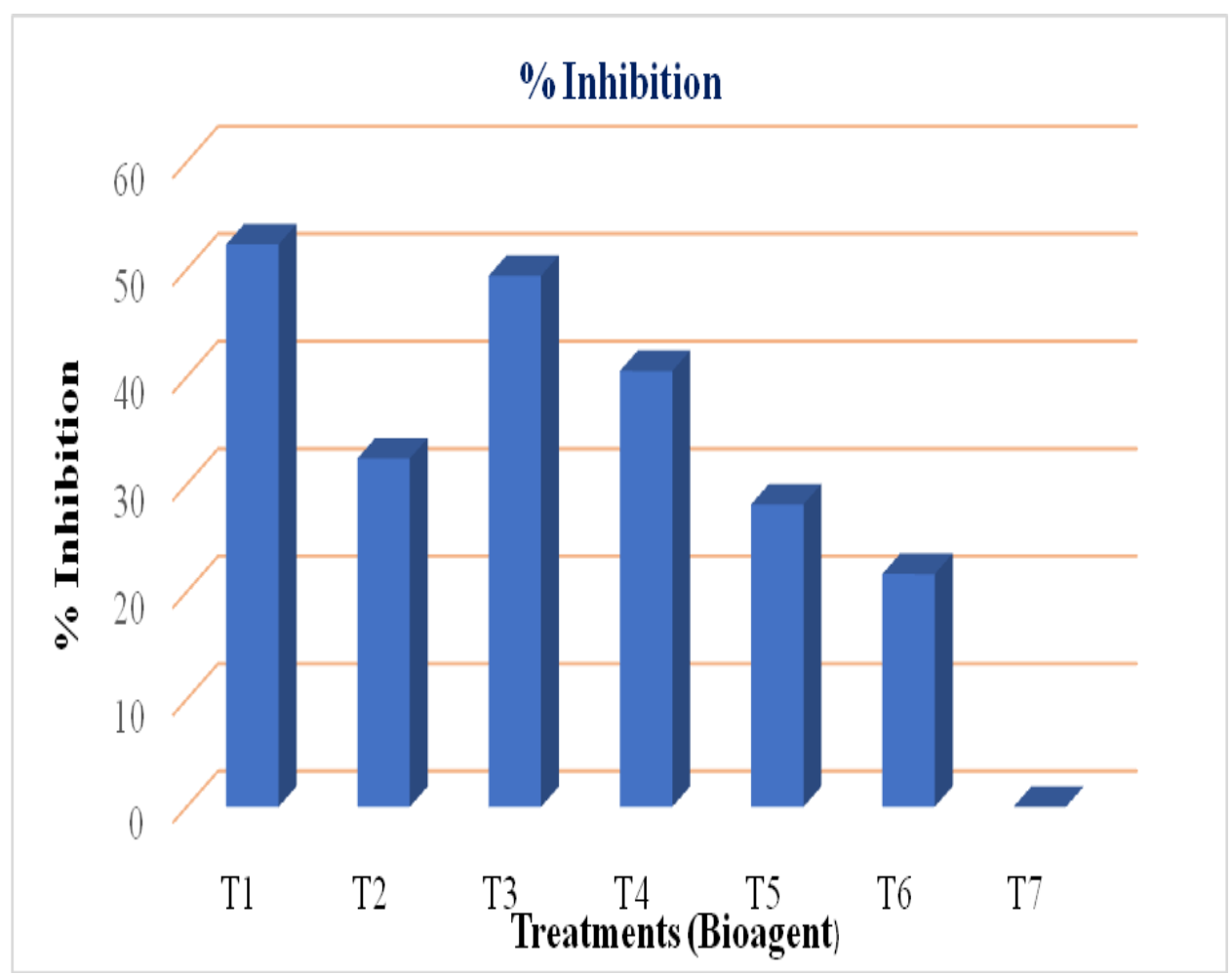


Fig.4 In vitro efficacy different bioagents against Fusarium oxysporum f. sp. lycopersici

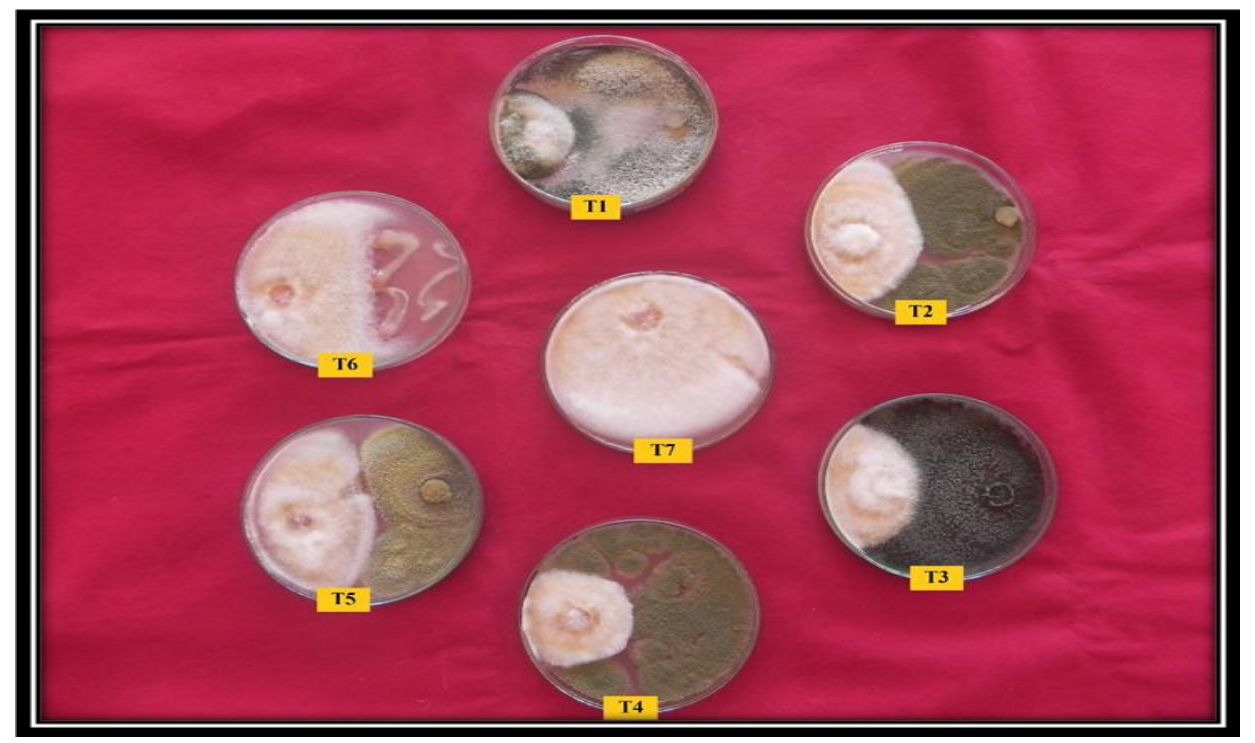

Evaluation of contact and combi fungicides against $F$. oxysporum f. sp. lycopersici

All of the four contact and three combi fungicides (Table 6) evaluated in vitro (each @ 1500, 2000 and 2500 ppm) were found fungistatic and significantly inhibited mycelial growth of $F$. oxysporum f. sp. lycopersici at all three test concentrations, over untreated control.

At $1500 \mathrm{ppm}$, mycelial growth inhibition of $F$. oxysporum f. sp. lycopersici ranged from 26.64 to 61.82 per cent. However, the contact fungicides viz., significantly highest mycelial inhibition $(61.82 \%)$ was recorded with the fungicide Copper oxychloride 50\% WP, followed by Thiram $75 \%$ WS (58.82\%), Captan 75\% WP (55.88\%) and Mancozeb 75\% WP (26.64\%), which was least effective.

At 1500 ppm, mycelial growth inhibition of $F$. oxysporum f. sp. lycopersici ranged from 27.74 to 100 per cent. However, the combi fungicides viz., significantly highest mycelial inhibition (100\%) was recorded with the fungicide Carbendazim 25\% + Mancozeb 50 $\%$ WS, followed by Carboxin $37.5 \%$
+Thiram 37.5\% WS(83.33\%) and Metalaxyl $8 \%$ + Mancozeb 64\% WP (27.74\%), which was least effective.

At 2000 ppm, mycelial growth inhibition of $F$. oxysporum f. sp. lycopersici ranged from 47.00 to 66.48 per cent. However, the contact fungicides viz., significantly highest mycelial inhibition (66.48\%) was recorded with the fungicide, Copper oxychloride 50\% WP, followed by Captan 75\% WP (62.92\%), Thiram 75\% WS (61.48\%) and Mancozeb $75 \%$ WP (47.00\%) which was less effective.

At 2000 ppm, mycelial growth inhibition of $F$. oxysporum f. sp. lycopersici ranged from 38.85 to 100 per cent. However, the combi fungicides viz., significantly highest mycelial inhibition (100\%) was recorded with the fungicide Carbendazim 25\% + Mancozeb 50 $\%$ WS, followed by Carboxin $37.5 \%$ +Thiram 37.5\% WS(86.66\%) and Metalaxyl $8 \%$ + Mancozeb 64\% WP (38.85\%), which was least effective.

At $2500 \mathrm{ppm}$, test contact fungicides exhibited similar trend but with increased mycelial growth inhibition as compared to 
that of at $1500 \mathrm{ppm}$ and $2000 \mathrm{ppm}$ and it was ranged from 47.72 to 67.37 per cent, However, the contact fungicides viz, significantly highest mycelial growth inhibition (67.37\%) was recorded with the fungicide Copper oxychloride 50\% WP, followed by Captan 75\% WP (64.01\%), Thiram 75\% WS (63.66\%) and Mancozeb $75 \%$ WP $(47.72 \%)$ which was less effective.

At $2500 \mathrm{ppm}$, test combi fungicides exhibited similar trend but with increased mycelial growth inhibition as compared to that of at $1500 \mathrm{ppm}$ and $2000 \mathrm{ppm}$ and it was ranged from 61.09 to 100 per cent, However, the combi fungicides viz., significantly highest mycelial growth inhibition (100\%) was recorded with the fungicide Carbendazim $25 \%$ + Mancozeb $50 \% \mathrm{WS}$, followed by Carboxin $37.5 \%$ +Thiram 37.5\% WS $(88.51 \%)$ and Metalaxyl $8 \%+$ Mancozeb $64 \%$ WP (61.09\%), which was least effective.

\section{Evaluation of bioagents}

The test biocontrol agents significantly inhibited mycelial growth of $F$. oxysporumf. sp. lycopersici, over untreated control. However, T. harzianum was found most effective with significantly least mycelial growth $(42.90 \mathrm{~mm})$ and it's highest inhibition (52.33\%), followed by $T$. virens $(45.53 \mathrm{~mm}$ and $49.41 \%$, respectively), $T$. koningii (53.50 $\mathrm{mm}$ and $40.55 \%$, respectively.), $T$. viride (60.83 $\mathrm{mm}$ and $32.41 \%$, respectively), $T$. hamatum $(64.67 \mathrm{~mm}$ and $28.14 \%$ respectively), $P$. fluorescens $(70.50 \mathrm{~mm}$ and $21.66 \%$, respectively) (Fig. 4).

These results are in conformity with the earlier findings of Barari H. (2015) who reported Trichoderma harzianum as most effective against $F$. oxysporum f. sp. lycopersici causing wilt disease in tomato crop. Similarly, Hegd, et al., (2017), Malathi (2015), and Mishra et al., (2017) reported the efficacy of $T$. harzianum against $F$. oxysporum, causing wilt disease in safflower, tomato, fir, tomato, onion and chilli crops. These results are in conformity with the earlier findings of Rudresh et al., (2005), who reported Trichoderma virens as most effective against $F$. oxysporum f. sp. ciceris causing wilt disease in Chick pea crop. Similarly, Govindappa et al., (2010), Gupta (2016).

In conclusion the various fungicides and bioagents are evaluated in vitro by applying poisoned food technique by using Potato Dextrose Agar as basal medium. In case of fungicide they were found effective in reduction of mycelial growth.

All the treatments used in this were significantly highest and cent per cent mycelial growth inhibition (100\%) was recorded with the fungicides viz., Carbendazim 50\% WP resulted with $(00.00$ $\mathrm{mm})$ mycelial growth followed by Copper oxychloride 50\% WP resulted with (31.47 $\mathrm{mm})$ mycelial growth and Carbendazim $25 \%$ + Mancozeb $50 \%$ WS resulted with $(00.00$ $\mathrm{mm})$ mycelial growth.

In case of bio-agent the results revealed that all of the test biocontrol agents significantly inhibited mycelial growth of $F$. oxysporum $\mathrm{f}$. sp. lycopersici, over untreated control. However, T. harzianum was found most effective with significantly least mycelial growth $(42.90 \mathrm{~mm})$ and it's highest inhibition (52.33\%), followed by $T$. virens $(45.53 \mathrm{~mm}$ and $49.41 \%$, respectively).

\section{References}

Anonymous (2017).National Horticultural Board Database, India.

Agrios, G.N. (1988). Studied the symptoms of Fusarium oxysporum Pl. Pathol, $\left(3^{\text {rd }}\right.$ Edn) Academic Press, New York. pp. 803. Altinok, H.H. (2005). First report of Fusarium wilt of egg plant, caused by Fusarium 
oxysporum f. sp. melongenae, in Turkey. Pl. Pathol.54: 577.

Ayed F., Majda D. R., Hayfa J. K. and Mohamed El Mahjoub.(2007). In vitro and in vivo evaluation of some biofungicides for potato Fusarium wilt biocontrol. Int. J. of Agri. Res. 2: 282288.

Barari H. (2016). Biocontrol of tomato Fusarium wilt by Trichoderma species under in vitro and in vivo conditions. Cercetari Agronomiceîn Moldova. 19 (165): 91-98.

Bashar, M. A.; Akter, A. and Hossain, K.S. (2015). Potential fungicides and plant extracts against Fusarium wilt of Brinjal. Dhaka Univ. J. Biol. Sci. 24(2): 209-213.

Bhimani, M.D.; Golakiya B.B. and Akbari L.F. (2018).Evaluation of different fungicides against fenugreek wilt (Fusarium oxysporum Schlecht.) I.J.C.S.6(2): 29-34.

Bhadra, M.; Khair, A.; Hossain, M.A.; Shamoli, F.A. and Sikder M.M. (2016). Biological Control of Wilt of Eggplant Caused by Fusarium solani f. sp. melongenae. Int. J. Expt. Agric. 6 (2): 20-25.

Dar W. A., Beig M. A., Ganie S. A., Bhat J. A., Shabir-u-Rehman and Razvi S. M. (2013). In vitro study of fungicides and biocontrol agents against Fusarium oxysporum $\mathrm{f}$. sp. pini causing root rot of Western Himalayan fir (Abiespindrow). Sci. Res. Essays. 8(30): 1407-1412.

Dahal N. and Shrestha R. K. (2018).Evaluation of efficacy of fungicides against Fusarium oxysporum f. sp. lentisin vitro at Lamjung, Nepal. J. Inst. Agric. Anim.
Sci. 35: 105-112.

Dennis, K.L. and Webster, J. (1971). Antagonistic properties of species group of Trichoderma and hyphal interaction. Trans.British Mycol. Soc.57:363-396.

Dubey, S.C.; M. Suresh.and Singh, B. (2007). Evalution of Trichodermaspecies against Fusarium oxysporumf.sp.ciceri for integrated management of chikpea wilt. Biol. Cont. 40 (1):118-127.

Gupta V.K. and Misra A.K. (2009).Efficacy of bioagents against Fusarium wilt of guava. J Myco/P/Patho/39(1):101-106.

Govindappa M., Ravishankar V. and Lokesh S. (2010). In vitro and In vivo responses of different treating agents against wilt disease of safflower. Journal of Cereals and Oilseeds.2 (1): 16-25.

Golakiya, B.B; Bhimani, M.D.; and Akbari L.F (2018).Efficacy of different fungicides for the management of chickpea wilt (Fusarium oxysporum f. sp. ciceri). I.J.C.S.6(2): 199-205.

Ghante, P.H.; Apet, K.T.; Kanase K.M.; Daunde A.T. and P.G. Chavan.(2018). In vitro efficacy of fungicides against Fusarium oxysporum f. sp. udum causing wilt disease of pigeonpea. Journal of Pharmacognosy and Phytochemistry 2019; 8(1): 1927-1931.

Hegde K. T., Narayanaswamy H., Kavitha S. Veeraghanti and Manu T. G. (2017).Efficacy of bio-agents, botanicals and fungicides against Fusarium oxysporum f. sp. dianthi causing wilt of carnation. Int. J. of Chemical Studies. 5(6): 139-142.

\section{How to cite this article:}

Gadhave, A. D., P. D. Patil, M. B. Dawale, A. P. Suryawnshi, M. S. Joshi and Giri, V. V. 2020. In-vitro Evaluation of Different Fungicides and Bioagents against Fusarium oxysporum f. sp. lycopersici. Int.J.Curr.Microbiol.App.Sci. 9(08): 3576-3584.

doi: https://doi.org/10.20546/ijcmas.2020.908.412 